\title{
Nature of Radiative Recombination Processes in Layered Heterogeneous PbCdI 2 Thick Films: Promising Scintillator Materials
}

\author{
Anatolii P. Bukivskii, ${ }^{1}$ Yuriy P. Gnatenko ${ }^{1},{ }^{1}$ Yuri P. Piryatinski, ${ }^{1}$ Igor V. Fesych, ${ }^{2}$ \\ Vasyl V. Lendel, ${ }^{2}$ Vasyl M. Tkach, ${ }^{3}$ and Petro M. Bukivskij ${ }^{1}$ \\ ${ }^{1}$ Institute of Physics, NASU, Prospect Nauky 46, Kyiv 03028, Ukraine \\ ${ }^{2}$ Taras Shevchenko National University of Kyiv, 64 Volodymyrska Str., Kyiv 01601, Ukraine \\ ${ }^{3}$ Institute for Superhard Materials, NASU, 2 Avtozavodska Str., Kyiv 04074, Ukraine \\ Correspondence should be addressed to Yuriy P. Gnatenko; yuriygnatenko@ukr.net
}

Received 26 December 2017; Revised 20 February 2018; Accepted 19 March 2018; Published 2 May 2018

Academic Editor: Jan A. Jung

Copyright ( 2018 Anatolii P. Bukivskii et al. This is an open access article distributed under the Creative Commons Attribution License, which permits unrestricted use, distribution, and reproduction in any medium, provided the original work is properly cited.

For the first time, $\mathrm{PbCdI}_{2}$ alloys in the form of thick films were prepared on a glass substrate using the conventional one-step chemical deposition method. The studies of the structural properties of these films showed that they have a very complex crystal structure, where $\mathrm{PbI}_{2}$ microcrystallites of micron and submicron sizes, as well as small nanoclusters (NCLs), are randomly formed in the $\mathrm{CdI}_{2}$ crystal matrix. It was found that the films show intense photo- and cathodoluminescence at room temperature. The temperature dependence of PL spectra of thick $\mathrm{PbCdI}_{2}$ films was studied. The nature of various radiative recombination processes in $\mathrm{PbCdI}_{2}$ films was established and it was shown that their PL spectra at room temperature are equally determined both by surface states in small $\mathrm{PbI}_{2}$ NCLs and by intrinsic defects in microcrystallites. The obtained $\mathrm{PbCdI}_{2}$ thick films can be considered as novel promising semiconductor materials for the development of effective inexpensive scintillator detectors for biomedical and industrial applications.

\section{Introduction}

Bulk nanostructured materials, that is, solids with nanoscale or partly nanoscale microstructures, are of great interest because of their unique physical properties $[1,2]$. It is possible to obtain these materials with required electronic parameters suitable for their efficient application in modern optoelectronics. An important place among these materials is occupied by layered materials characterized by strong ioncovalent bonds within the layers and weak van der Waals bonds between them. In the case of nanostructured layered materials, they can be considered as two-dimensional (2D) layered nanomaterials, such as graphene and germanene [3].

Layered $\mathrm{PbI}_{2}$ semiconductors are promising materials, since they have a high potential for the development of noncooled detectors for both $\gamma$ - and X-radiation [4-8]. Recently, it was shown that the alloys based on $\mathrm{PbI}_{2}$, namely, $\mathrm{PbCdI}_{2}$ crystals, are nanostructured bulk solids, where $\mathrm{PbI}_{2}$ nanoclusters (NCLs) are naturally formed in $\mathrm{CdI}_{2}$ matrix during the growth process [9-14]. The bulk $\mathrm{PbI}_{2}$ crystal is a direct bandgap semiconductor with an energy gap of about $2.5 \mathrm{eV}$ [15-17]. It was found that the $\mathrm{Pb}_{0.30} \mathrm{Cd}_{0.70} \mathrm{I}_{2}$ crystals are unusual solid solutions with randomly distributed $\mathrm{PbI}_{2}$ NCLs of different sizes, including also NCLs of small sizes (several $\mathrm{nm}$ ), which exhibit a quantum confinement effect for free excitons. Their emission can be observed in the spectral range of 2.7-3.0 eV. Thus, the heterogeneous nanostructured $\mathrm{Pb}_{0.30} \mathrm{Cd}_{0.70} \mathrm{I}_{2}$ solid solutions have crystal regions with $\mathrm{PbI}_{2}$ NCLs having different band gaps. It should be noted that the direct band gap of the $\mathrm{CdI}_{2}$ matrix corresponds to the value characteristic of bulk crystals and equals $3.6 \mathrm{eV}$. Therefore, the $\mathrm{CdI}_{2}$ matrix is transparent for the emission of both large and small $\mathrm{PbI}_{2}$ NCLs. It was established that the bulk $\mathrm{Pb}_{0.30} \mathrm{Cd}_{0.70} \mathrm{I}_{2}$ alloys are very promising scintillator 
materials and have strong photoluminescence (PL) and X-ray luminescence due to the presence of small $\mathrm{PbI}_{2}$ NCLs [13].

In this work, we study structural, morphological, and $\mathrm{PL}$ properties of $\mathrm{PbCdI}_{2}$ thick films, prepared by the conventional one-step chemical deposition method. In addition, an X-ray dispersion analysis of the local chemical composition of the investigated films was also carried out. The aim of these studies is to establish the nature of different radiative recombination processes in this material and to compare it with that characteristic of bulk nanostructured $\mathrm{PbCdI}_{2}$ solid solutions. Understanding these processes is an important issue that is of both physical and technological interest, since these films can be considered as novel effective inexpensive scintillator materials. It is also expected that such films, analogously to bulk crystals, are highly heterogeneous nanostructured materials and, therefore, are of considerable interest for condensed matter physics.

\section{Materials and Methods}

The $\mathrm{Pb}_{1-x} \mathrm{Cd}_{x} \mathrm{I}_{2}(X=0.70)$ films were prepared by the conventional one-step deposition method [18]. To obtain the $\mathrm{Pb}_{0.30} \mathrm{Cd}_{0.70} \mathrm{I}_{2}$ precursor solution with a molar ratio $\mathrm{PbI}_{2}$ : $\mathrm{CdI}_{2}=30: 70,138 \mathrm{mg}(0.3 \mathrm{mmol})$ of lead iodide $\left(\mathrm{PbI}_{2}, 99.9 \%\right)$ and $256 \mathrm{mg}(0.7 \mathrm{mmol})$ of cadmium iodide $\left(\mathrm{CdI}_{2}, 99.9 \%\right)$ were dissolved in $2 \mathrm{ml}$ of absolute $\mathrm{N}, \mathrm{N}$-dimethylformamide (DMF, $\geq 99 \%$ ). Such proportions of components are optimal, since increasing the mass of iodides in $2 \mathrm{ml}$ of DMF leads to the formation of a precipitate. The reaction mixture was treated in the ultrasonic bath (InterSonic IS-4, Poland) for $10 \mathrm{~min}$. Then, the clear bright yellow precursor solution was spin-coated on a well-cleaned glass substrate (3.0 by $3.0 \mathrm{~cm}$ ) at a speed of $500 \mathrm{rpm}$ for $30 \mathrm{~s}$ and dried at $90 \mathrm{C}$ for $30 \mathrm{~min}$. After drying the lead-cadmium iodide films, they were annealed in air at $150 \mathrm{C}$ for $5 \mathrm{~min}$ to remove the remaining DMF. The thickness of the $\mathrm{PbCdI}_{2}$ films was about $5-10 \mu \mathrm{m}$. It should be noted that all the procedures were performed in open-air at room temperature.

The crystal quality and presence of different phases in $\mathrm{PbCdI}_{2}$ films were verified by X-ray diffraction experiments (XRD) using a Shimadzu LabX XRD-6000 diffractometer with $\mathrm{Cu} \mathrm{K}_{\alpha}$ radiation $(\lambda=0.154178 \mathrm{~nm})$. Measurements were carried out over the scattering angle range of $2 \Theta=$ $10-60^{\circ}$ with a step of $0.02^{\circ}$. XRD analysis was carried out using the ICDD database, PDF-2 Release 2012.

The surface structure of the films was studied using a Zeiss EVO 50 XVP scanning electron microscope with an $\mathrm{X}$-ray dispersion analysis module in two regimes: phasecontrast and SE + visual. This allowed us not only to obtain a visualization of the film structure morphology, but also to reveal areas that show cathodoluminescence of different intensity, as well as determining the chemical composition of such areas. Thus, this made it possible to observe precisely the areas where $\mathrm{PbI}_{2}$ NCLs were formed in the $\mathrm{PbCdI}_{2}$ solid solutions.

The study of the steady state PL spectra of the investigated films at room and liquid helium temperatures, as well as the measurement of their temperature dependencies, was performed using a MAYA-2000PRO spectrometer [11, 13]. The temperature was stabilized using a UTREX system connected to a variable temperature liquid helium cryostat. Temperature fluctuations in this case were less than $0.01 \mathrm{~K}$. The PL spectra were excited by emission of an LED with $\lambda=395 \mathrm{~nm}$ and the power of $20 \mathrm{~mW}$. Uniform illumination of the sample surface was provided using an optical fiber with a diameter of $600 \mu \mathrm{m}$. Mathematical analysis of experimental PL spectra was carried out using the Peak Analyzer module of the OriginPro software, which allows us to resolve individual bands of each PL spectrum and analyze temperature dependencies of some parameters of these bands, such as peak position and intensity.

\section{Results and Discussion}

3.1. Characterization of $\mathrm{PbCdI} \mathrm{I}_{2}$ Alloys. Both $\mathrm{PbI}_{2}$ and $\mathrm{CdI}_{2}$ are layered semiconductor crystals that are formed by repeated layers of I-Pb-I or I-Cd-I, respectively $[19,20]$. These materials can crystallize in hexagonal and rhombohedral structures. Today, several tens of their polytypes are known. It should be noted that main basic types of crystalline polytypic modifications are $2 \mathrm{H}, 4 \mathrm{H}$, and $12 \mathrm{R} .2 \mathrm{H}$ and $4 \mathrm{H}$ are the most stable polytypes which can be grown at room temperature. They act as basic structural units for the formation of higher polytypes. $12 \mathrm{R}$ also has a stable structure; however, it usually forms at higher temperatures. The lattice parameters of $\mathrm{PbI}_{2}$ and $\mathrm{CdI}_{2}$ are very close. Therefore, solid solutions based on these crystals are expected to form relatively easy. However, the $\mathrm{PbCdI}_{2}$ alloy is a nonisoelectronic system, since the valence electrons of $\mathrm{Pb}$ and $\mathrm{Cd}$ atoms belong to different electronic configurations, namely, $5 \mathrm{~d}^{10} 6 \mathrm{~s}^{2} 6 \mathrm{p}^{2}$ and $4 \mathrm{~d}^{10} 5 \mathrm{~s}^{2}$. We assume that nonisoelectronic substitution of cation sites is a reason for the formation of $\mathrm{PbI}_{2}$ NCLs of different sizes in $\mathrm{CdI}_{2}$ matrix during the growth of $\mathrm{PbCdI}_{2}$ crystals using the Bridgman method [11]. Besides, in our opinion, such a behavior could also be caused by spinodal decomposition of these solid solutions [21-24]. Thus, these materials are strongly heterogeneous solids and have a very complex crystal structure.

The XRD spectrum of $\mathrm{PbCdI}_{2}$ films, grown by the conventional one-step deposition method, is shown in Figure 1. The presence of sharp diffraction peaks means that the material being studied is crystalline rather than amorphous. Analysis of XRD spectra indicates that the investigated alloys include different crystal phases of both $\mathrm{CdI}_{2}$ and $\mathrm{PbI}_{2}$ crystals. In particular, here we observe three polytypes of $\mathrm{CdI}_{2}: 2 \mathrm{H}, 4 \mathrm{H}$, and $12 \mathrm{R}$. In the case of $2 \mathrm{H}$ polytype, the main (very intense) observed peaks are associated with the (001), (002), and (004) planes and all of them correspond to the [001] crystal direction. It should be noted that the (001) plane is perpendicular to the crystal c-axis. The other intense minor peaks were identified as related to the (100), (011), (012), (003), (110), and (202) planes. For $4 \mathrm{H}$ polytype, the main observed peaks were associated with the (002), (004), and (008) planes. At the same time, other low intensity peaks are also observed, which can be identified with the (102), (006), (101), and (008) planes. Therefore, we can assume that $\mathrm{CdI}_{2}$ of $2 \mathrm{H}$ and $4 \mathrm{H}$ polytypes are oriented in the [100] preferred direction. The intensity of the peaks associated with the 12R polytype of 


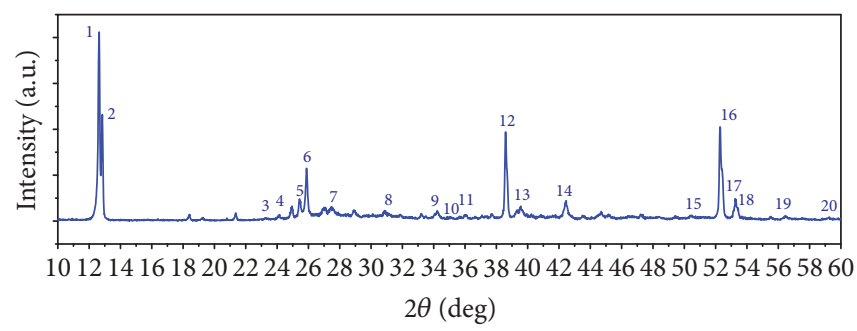

FIgURE 1: XRD spectrum of $\mathrm{PbCdI}_{2}$ thick film.

TABLE 1: Chemical composition of $\mathrm{PbCdI}_{2}$ film, obtained for spots 1-13, shown in Figure 2(a).

\begin{tabular}{|c|c|c|c|c|c|}
\hline Spots & $\mathrm{C}(\%)$ & $\mathrm{O}(\%)$ & $\mathrm{Cd}(\%)$ & $\mathrm{I}(\%)$ & $\mathrm{Pb}(\%)$ \\
\hline 1 & 1.79 & 1.85 & 12.55 & 59.66 & 24.16 \\
\hline 2 & 3.75 & 1.34 & 9.93 & 56.79 & 28.19 \\
\hline 3 & 3.76 & 1.07 & 2.39 & 54.67 & 38.11 \\
\hline 4 & 2.68 & 0.14 & 7.07 & 58.42 & 31.69 \\
\hline 5 & 7.71 & 4.42 & 26.53 & 59.71 & 1.64 \\
\hline 6 & 8.81 & 5.76 & 25.57 & 58.47 & 1.40 \\
\hline 7 & 12.21 & 5.12 & 23.96 & 57.19 & 1.52 \\
\hline 8 & 4.30 & 1.88 & 28.62 & 64.05 & 1.15 \\
\hline 9 & 2.29 & 1.43 & 29.53 & 66.48 & 0.27 \\
\hline 10 & 3.13 & 0.98 & 28.47 & 66.12 & 1.30 \\
\hline 11 & 0.60 & 0.67 & 30.03 & 68.07 & 0.62 \\
\hline 12 & 2.78 & 2.36 & 28.33 & 65.15 & 1.38 \\
\hline 13 & 0.99 & 2.37 & 28.04 & 66.51 & 2.08 \\
\hline
\end{tabular}

$\mathrm{CdI}_{2}$, namely, (006), (101), (104), (0111), (0114) (110), (024), and (2014), is low compared to the intensity of main peaks of $2 \mathrm{H}$ and $4 \mathrm{H}$ polytypes. At the same time, two most intense peaks associated with the (006) and (110) planes can be noted among them. This indicates that, for this crystal modification, there are two preferred directions; that is, the crystal layers grow mainly in perpendicular or parallel directions to the substrate surface. It should be noted that the presence of the other peaks shows that there are also other directions of the c-axis, which are inclined to the substrate surface. Since the peaks associated with $12 \mathrm{R}$ polytype have very low intensity in comparison with that for $2 \mathrm{H}$ and $4 \mathrm{H}$ polytypes, their relative concentration in $\mathrm{PbCdI}_{2}$ alloys is small. It should be noted that earlier the formation of 12R polytype was observed in $\mathrm{CdI}_{2}$ crystal doped with $\mathrm{Pb}$ [25].

The XRD analysis showed that the investigated $\mathrm{PbCdI}_{2}$ alloys also contain $\mathrm{PbI}_{2}$ crystal phases of $2 \mathrm{H}$ and $4 \mathrm{H}$ modifications. In this case, the most intense peaks in XRD spectrum were identified as related to the (002), (006), and (008) planes of $\mathrm{PbI}_{2}$ of $4 \mathrm{H}$ polytype. It should be noted that other peaks of lower intensity related to the (101), (004), (102), (012), (104), (110), (204), and (116) planes are also observed. The peak associated with (110) plane corresponds to the direction of caxis perpendicular to the substrate surface. We assume that such $\mathrm{PbI}_{2}$ microcrystals or NCLs are formed in $\mathrm{CdI}_{2}$ of $2 \mathrm{H}$ or $12 \mathrm{R}$ polytypes because they have an analogous peak related to this crystal plane. For $2 \mathrm{H} \mathrm{PbI}_{2}$ the observed intense peak can be associated with (011) plane and other observed peaks of low intensity are related to the (012), (110), and (104) planes.
This indicates that the preferred direction of crystal layers for $2 \mathrm{H} \mathrm{PbI}_{2}$ is the [011] direction. The other (100), (012), and (001) peaks of low and very low intensity are also observed. At the same time, for the $4 \mathrm{H} \mathrm{PbI}_{2}$ phase the growth of the layers parallel to the substrate surface is the most probable.

The results of a surface morphology study of $\mathrm{PbCdI}_{2}$ thick films performed using a Zeiss EVO 50 XVP scanning electron microscope with an X-ray dispersion analysis module performed at room temperature are shown in Figure 2(a). The surface morphology of the studied films was obtained in phase-contrast mode. In this case, there are areas with different brightness. Therefore, it is very important to determine local chemical composition of the film for different surface spots. These results are shown in Figure 2(b) and Table 1.

As can be seen from Figure 2(b), a chemical analysis for spot 1 , which is one of the brightest areas, shows that it contains a high concentration of $\mathrm{Pb}(24.16 \%)$. At the same time, the concentration of $\mathrm{Cd}$ is only $12.55 \%$; that is, this area is enriched in $\mathrm{Pb}$. There are also other bright areas shown in Figure 2(a), in particular, spots 2 and 3, which according to the data presented in Table 1 are also enriched in $\mathrm{Pb}$ ( $28.19 \%$ and $38.11 \%$, resp.). It should be noted that visually the brightness of these areas is approximately the same. However, their chemical composition is different. It should be noted that in this case the depth of X-ray photons penetration is about 1.0 micron. Thus, we assume that different $\mathrm{Pb}$ and Cd concentrations for various spots can be caused by the inhomogeneous composition of the film both at the surface 


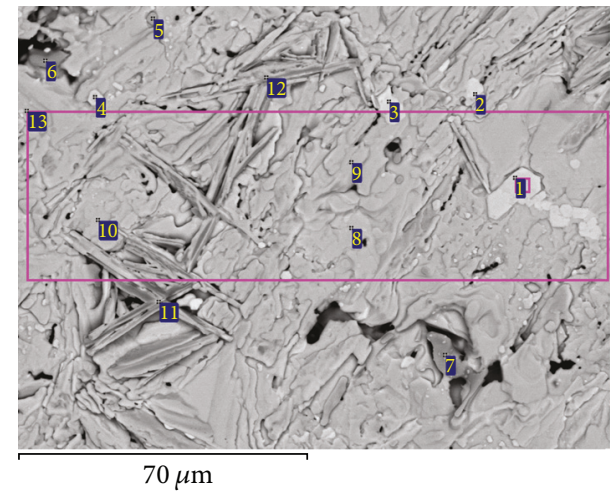

(a)

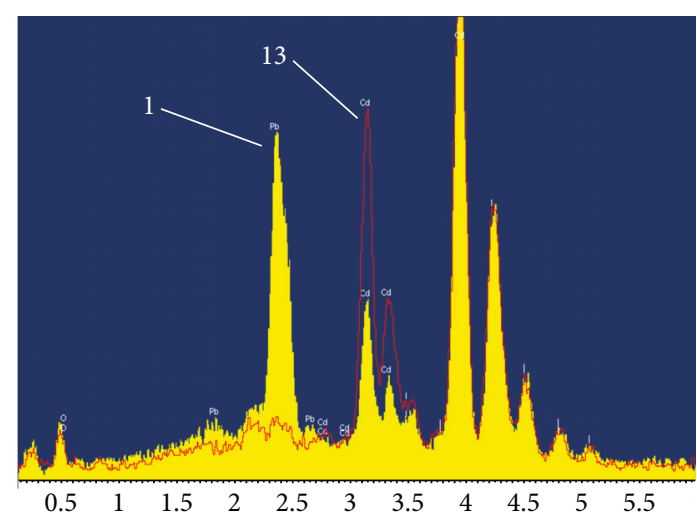

(b)

FIGURE 2: SEM images (a) and EDS spectrum (b) of $\mathrm{PbCdI}_{2}$ thick film obtained in phase-contrast mode using a Zeiss EVO 50 XVP scanning electron microscope. Spots 1-13 in SEM images indicate the areas of the film surface where its chemical composition has been determined. EDS spectra correspond to 1 and 13 spots, respectively.

TABLE 2: Chemical composition of $\mathrm{PbCdI}_{2}$ film, obtained for spots 1-7 shown in Figure 3(b).

\begin{tabular}{lccccc}
\hline Spots & $\mathrm{C}(\%)$ & $\mathrm{O}(\%)$ & $\mathrm{Cd}(\%)$ & $\mathrm{I}(\%)$ & 56.61 \\
\hline 1 & 3.89 & 1.78 & 13.63 & 59.18 & 24.08 \\
2 & 2.23 & 2.68 & 18.51 & 66.30 & 17.41 \\
3 & 1.28 & 2.17 & 28.73 & 70.48 & 1.51 \\
4 & 1.27 & 25.37 & 66.19 & 2.28 \\
5 & 2.60 & 2.34 & 28.68 & 65.81 & 0.29 \\
6 & 2.51 & 2.23 & 28.89 & 66.64 & 2.40 \\
7 & 0.67 & 1.75 & 28.44 & 1.99 \\
\hline
\end{tabular}

and in the volume; that is, they are the result of the existence of $\mathrm{PbI}_{2}$ and $\mathrm{CdI}_{2}$ layers of different thickness located parallel to the substrate surface. It is obvious that the existence of $\mathrm{PbI}_{2}$ microcrystallites for these spots is preferential. Figure 2(b) also shows the EDS spectrum for spot 13, which is typical of this film gray color in the SEM image. The average concentration of $\mathrm{PbI}_{2}$ for this spot is $2.08 \%$. Such a low $\mathrm{Pb}$ concentration is characteristic of different areas of the surface of the investigated films, excluding bright regions where $\mathrm{PbI}_{2}$ microcrystallites embedded in $\mathrm{CdI}_{2}$ matrix are mainly formed. The presence of regions with a low concentration of $\mathrm{Pb}$ can be caused by the possible formation of $\mathrm{PbI}_{2} \mathrm{NCLs}$ of different sizes in the same way as we have observed for bulk $\mathrm{Pb}_{0.30} \mathrm{Cd}_{0.70} \mathrm{I}_{2}$ alloys grown by the Bridgman method [11]. In the left side of Figure 2(a), the dark gray rods are observed which can be associated with the presence of $\mathrm{CdI}_{2}$ microcrystals with crystal layers oriented perpendicular or inclined to the substrate surface. These rods are generally arranged at an angle of 120 degrees to each other and have a low density due to the presence of intergranular voids. Taking into account the results of the XRD measurements, we assume that formation of such microcrystals can be caused by the formation of $\mathrm{CdI}_{2}$ polytype of $12 \mathrm{R}$ modification. As can be seen from Table 1 , the investigated films also contain $\mathrm{C}$ and $\mathrm{O}$ elements in low concentrations. We suppose that the presence of these elements is due to the features of the technological process of preparation of the films.
Figure 3 shows the SEM images obtained by scanning in the mode of phase-contrast (a) and visual photons and secondary electrons (b) registration. Taking into consideration the results shown in Table 2, we assume that the very bright hexagons, marked in Figure 3(a), correspond to $\mathrm{PbI}_{2}$ microcrystallites embedded in the $\mathrm{CdI}_{2}$ matrix. In Figure 3 (b) they look like dark spots due to the absence of cathodoluminescence from these areas. At the same time, the bright areas in Figure 3(b) may correspond to the film spots, where, in particular, small $\mathrm{PbI}_{2}$ NCLs may be embedded in the $\mathrm{CdI}_{2}$ matrix.

As we showed earlier [11, 13], in the case of bulk $\mathrm{Pb}_{0.30} \mathrm{Cd}_{0.70} \mathrm{I}_{2}$ solid solutions obtained by the Bridgman method, such crystal regions exhibit intense cathodoluminescence, as well as photoluminescence and X-ray luminescence at room temperature, and thus obviously look bright when scanning in the mode of visual photon registration of the SEM microscope.

It should be noticed that since the investigated films were obtained by drying a solution of $\mathrm{PbI}_{2}$ and $\mathrm{CdI}_{2}$ in absolute $\mathrm{N}, \mathrm{N}$-dimethylformamide, the residual solvent created a thin layer on the surface of the films interfering with direct observation of small $\mathrm{PbI}_{2}$ nanoparticles as it had been observed earlier for the bulk $\mathrm{PbCdI}_{2}$ crystals [14].

3.2. Nature of Radiative Recombination Processes in $\mathrm{PbCdI}_{2}$ Thick Films. More detailed information on the features of 


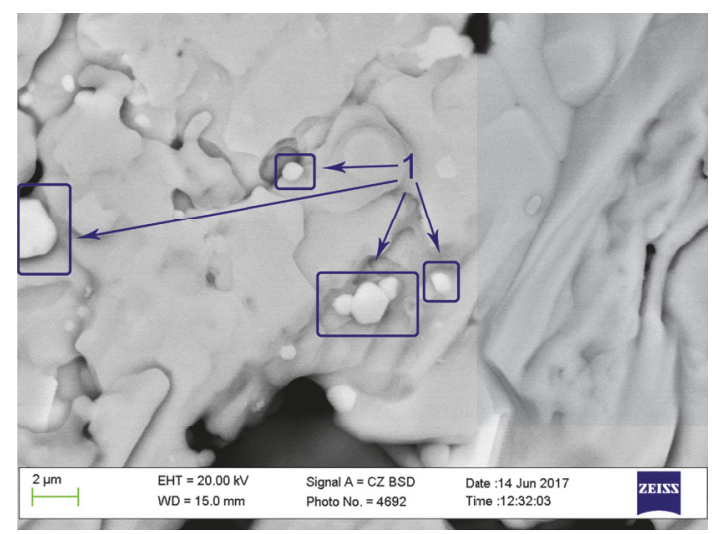

(a)

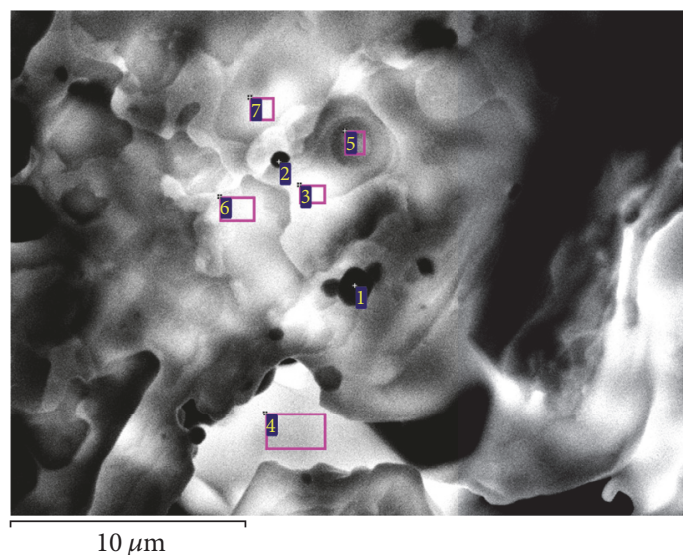

(b)

FIGURE 3: SEM images of $\mathrm{PbCdI}_{2}$ thick films obtained in phase-contrast mode (a) and VPSE mode (b). Very bright hexagons, marked in (a), correspond to microcrystallites of $\mathrm{PbI}_{2}$ embedded in the $\mathrm{CdI}_{2}$ matrix. The brightness of the areas presented in (b) corresponds to the intensity of their cathodoluminescence.

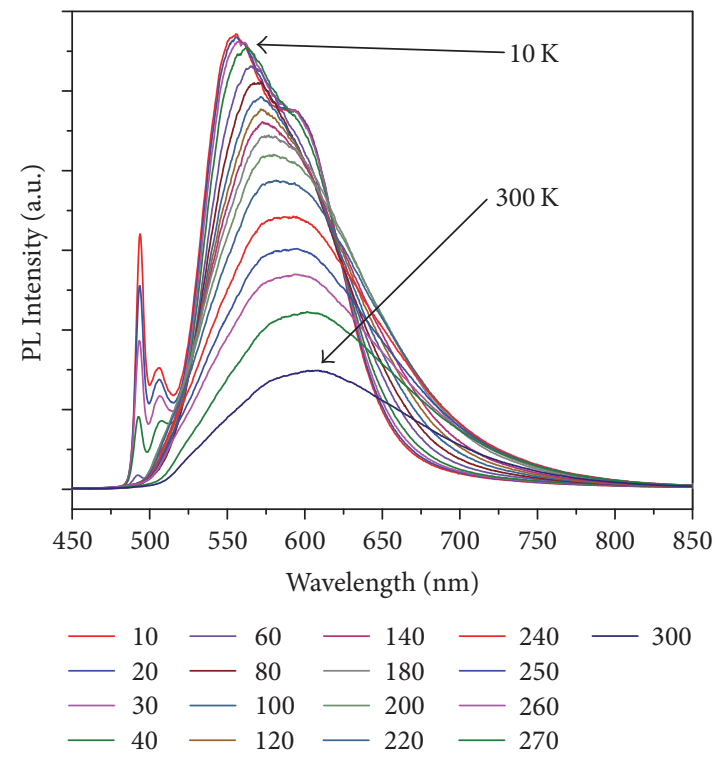

(a)

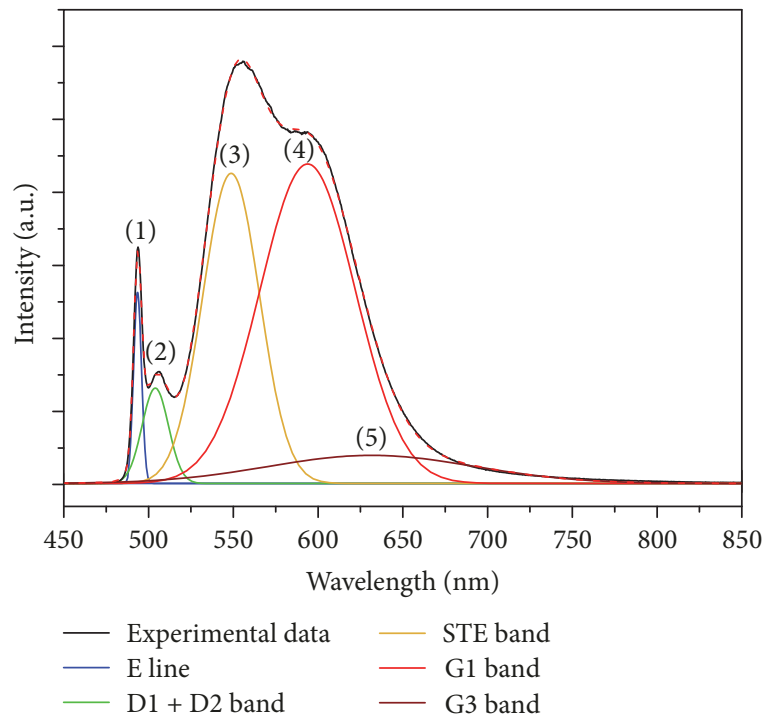

(b)

FIgURE 4: Temperature dependence of PL spectrum of $\mathrm{PbCdI}_{2}$ thick films (a). Fitting the separate bands that form $\mathrm{PL}$ spectrum of $\mathrm{PbCdI}{ }_{2}$ thick films at $T=10 \mathrm{~K}$. PL bands that correspond to the radiative recombination of bound and free excitons (E), recombination of donoracceptor pairs involving shallow acceptor centers (D1 + D2), recombination of self-trapped excitons (STE), and recombination of free electrons and the deep acceptor centers (G1 and G3) are observed (b). Curves 1-5 correspond to E, D1 + D2, STE, G1, and G3 bands, respectively. The red dashed curve represents the results of the fitting of the experimental data by the sum of Gaussian functions.

the crystalline structure of thick films can be obtained by studying the nature of their PL spectra. In addition, these studies also allow us to find ways to optimize the efficiency of their luminescence. The results of studying the temperature dependencies of the PL spectra of the investigated films are shown in Figure 4(a).

In order to study the nature of recombination processes in $\mathrm{PbCdI}_{2}$ films, mathematical analysis of the obtained spectra has been done using OriginPro software. The results of such analysis for the investigated $\mathrm{PbCdI}_{2}$ thick films at $10 \mathrm{~K}$ are shown in Figure 4(b). It was found that the observed PL spectra at different temperatures can be described by five components. As can be seen from Figure 4(b), intense emission (E line) is observed in short-wavelength spectral region at low temperatures. The narrow PL line at $4938 \AA$ $(2.511 \mathrm{eV})$ obviously corresponds to the emission of excitons bound on the neutral donors, that is, $\mathrm{D}^{\circ} \mathrm{X}$-line [26]. The energy position of this line is close to the exciton line (2.502 eV) observed for bulk $\mathrm{PbI}_{2}$ crystals of $4 \mathrm{H}$-modification [20]. In the case of $\mathrm{PbI}_{2}$ of $2 \mathrm{H}$ polytype the energy position 


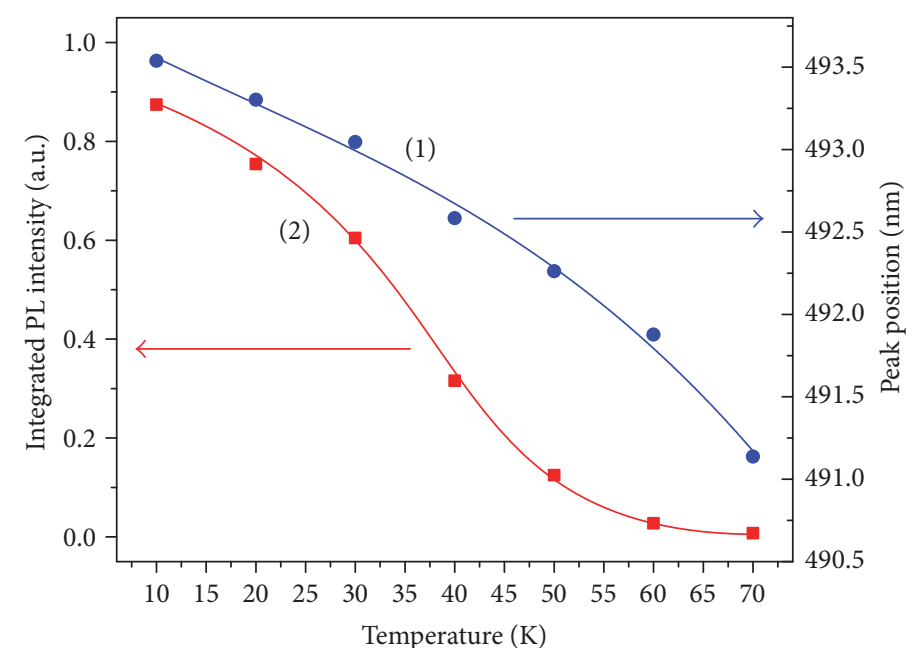

(a)

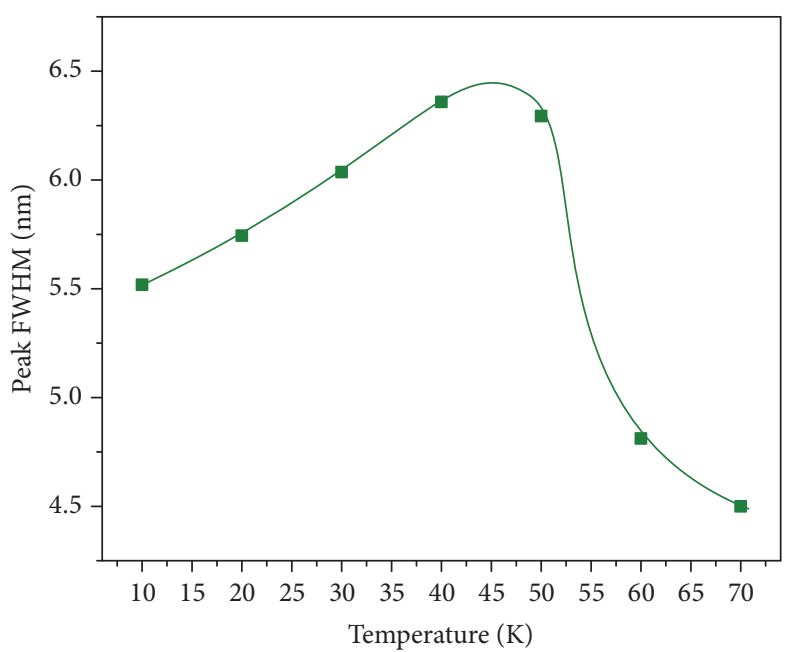

(b)

Figure 5: Dependence of exciton PL on temperature. (a) The temperature dependence of the integrated intensity and the peak position of PL exciton E band. (b) The temperature dependence of the FWHM value of E band.

of $\mathrm{D}^{\circ} \mathrm{X}$-line corresponds to $2.492 \mathrm{eV}$ [20]. The PL intensity of $\mathrm{D}^{\circ} \mathrm{X}$-line reduces with the increase in temperature.

The temperature dependence of energy position, full width at half maximum (FWHM), and integrated intensity of E line are shown in Figure 5. As can be seen from Figure 5(a), the energy position of this line is shifted to $4911 \AA(2.524 \mathrm{eV})$ at $T=70 \mathrm{~K}$. It should be noted that the energy position is determined by the emission of both bound and free excitons. The contribution of free excitons increases with temperature and becomes dominant at $T \geq 50 \mathrm{~K}$. At the same time, the $\mathrm{PL}$ intensity of free excitons increases. The energy position of free excitons $(2.524 \mathrm{eV})$ for the investigated films is markedly shifted to the high-energy side in comparison with that for bulk $\mathrm{PbI}_{2}$ crystals of $4 \mathrm{H}$ polytype $(2.508 \mathrm{eV})$ [20].

This means that the observed $\mathrm{E}$ line is caused by the exciton emission in both the microcrystallites and small NCLs which exhibit a weak quantum size effect. This assumption is supported by the measurements of the temperature dependence of the FWHM value of the exciton E line (Figure 5(b)), which initially increases with rising temperature up to about $50 \mathrm{~K}$ and then decreases abruptly at higher temperatures. This indicates that the emission of free excitons is a decisive factor in the temperature range above $50 \mathrm{~K}$. The strong decrease of the integrated intensity of the exciton line is also caused by the reduction of the bound exciton contribution with increasing temperature.

Another PL band at $5062 \AA(2.449 \mathrm{eV})$ coincides with the $\mathrm{PL}$ band in bulk $\mathrm{PbI}_{2}$ crystal $(2.441 \mathrm{eV})$, and corresponds to the recombination of donor-acceptor pairs (DAPs) [26, 27]. In this case, the PL band, named D1 band, is associated with stoichiometric defects at the interior of the $\mathrm{PbI}_{2}$ microcrystallites and nanoparticles [28-33]. It should be noted that for $\mathrm{PbI}_{2}$ bulk crystal another PL band, caused by the recombination of DAPs (D2 band), was also observed at $2.413 \mathrm{eV}$. This band is associated with a deeper acceptor center [27]. In the case of $\mathrm{PbCdI}_{2}$ films this $\mathrm{PL}$ band is practically not observed. The ionization energy of acceptor and donor centers associated with the emission of DAPs (D1 band) corresponds to the energy of $78 \mathrm{meV}$ and $110 \mathrm{meV}$, respectively [11].

As can be seen from Figure 4(b), the PL spectrum of $\mathrm{PbCdI}_{2}$ thick films also contains three broad bands, which determine their emission at higher temperatures $(T \geq 70 \mathrm{~K})$. The results of fitting the multiple peaks in PL spectra, shown in Figure 4(b) (curves 3-5), show the broad bands at $5484 \AA$ $(2.261 \mathrm{eV}), 5942 \AA(2.086 \mathrm{eV})$, and $6316 \AA(1.963 \mathrm{eV})$ at $10 \mathrm{~K}$. These PL bands determine the emission of $\mathrm{PbCdI}_{2}$ alloys in the spectral region from $5000 \AA(2.480 \mathrm{eV})$ to $8000 \AA$ $(1.550 \mathrm{eV})$ up to room temperature. It should be noted that the energy position of PL bands at $2.261 \mathrm{eV}$ and $2.086 \mathrm{eV}$ is close to that for bulk $\mathrm{Pb}_{0.30} \mathrm{Cd}_{0.70} \mathrm{I}_{2}$ nanostructured alloys $(2.291 \mathrm{eV}$ and $2.075 \mathrm{eV}$, resp.). Taking into account the results obtained by us in [11], we assume that the PL band at $2.261 \mathrm{eV}$ can be associated with emission of STE states. Formation, excitation, population, and dynamics of the STE states were experimentally studied for $\mathrm{Pb}_{0.30} \mathrm{Cd}_{0.70} \mathrm{I}_{2}$ crystals in our paper in detail [11] and their interpretation was based on the theory presented in [34]. Analysis of the temperature dependence of the PL band at $2.086 \mathrm{eV}$, named G1-band, showed that it can be associated with recombination of free electrons and acceptor centers ((e,A)-transitions). At the same time, the PL band at $1.963 \mathrm{eV}$ can be caused by a recombination process involving deeper acceptor centers [11]. In this case, recombination of free electrons and holes at these deep acceptor centers (the (e,A)-transitions) occurs.

This band was named G3 band in [11]. Taking into account the nature of the PL spectra of thick PbCdI2 films, optical transitions associated with various radiative recombination processes in films can be represented by the scheme shown in Figure 6. The comparison of these optical transitions with those of bulk $\mathrm{Pb}_{0.30} \mathrm{Cd}_{0.70} \mathrm{I}_{2}$ solid solutions indicates that their nature for both cases is the same. 


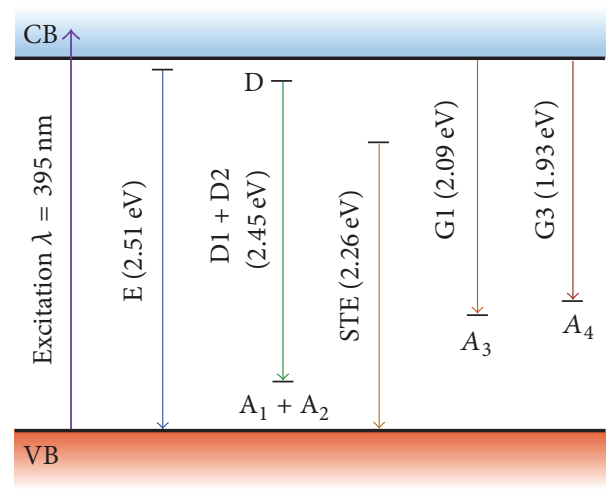

Figure 6: The energy scheme of the optical transitions which are associated with different radiative recombination processes in $\mathrm{PbCdI}_{2}$ thick films.

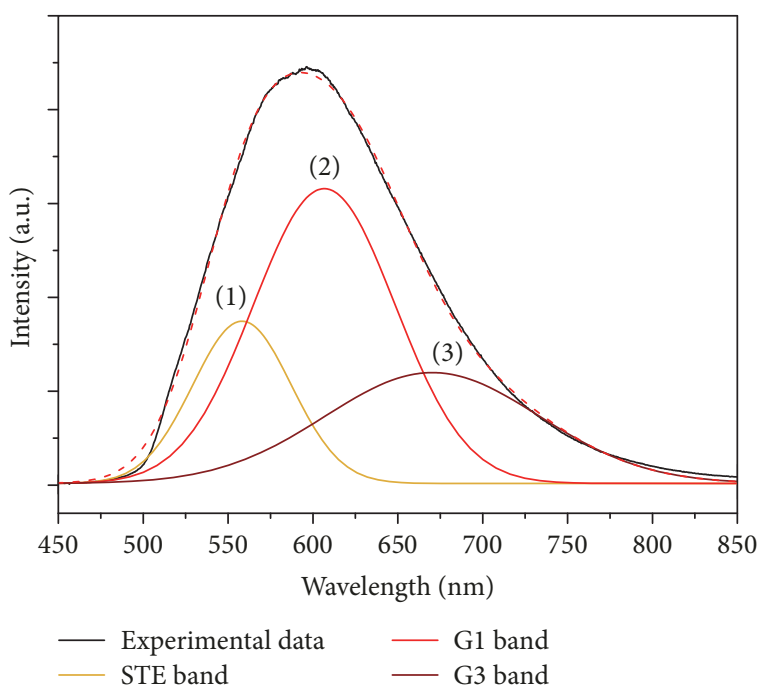

FIGURE 7: Fitting the separate PL bands that form the PL spectrum of $\mathrm{PbCdI}$ thick films at $T=300 \mathrm{~K}$. Curves $1-3$ correspond to STE, G1, and G3 bands, respectively. The red dashed curve represents the results of the fitting of the experimental data by the sum of Gaussian functions.

In Figure 7 the results of fitting the PL spectrum of the $\mathrm{PbCdI}_{2}$ thick films at room temperature are shown. It can be seen that the high temperature PL spectrum consists of three wide overlapping bands, namely, STE, G1, and G3 PL bands. Exciton photoluminescence and emission caused by the recombination of DAPs with participation of shallow acceptor levels do not appear in the PL spectrum, similarly to the case of bulk nanostructured $\mathrm{Pb}_{0.30} \mathrm{Cd}_{0.70} \mathrm{I}_{2}$ solid solutions [11].

The analysis of the temperature dependence of the PL spectra of $\mathrm{PbCdI}_{2}$ films presented in Figure 4(a) shows that the integral PL intensity decreases only about threefold with the increase in temperature from 4.5 to $300 \mathrm{~K}$, and it still remains significant at room temperature. It should be noted that PL intensity decreases mostly in the temperature range from 250 to $300 \mathrm{~K}$. Thus, the emission of the investigated
$\mathrm{PbCdI}_{2}$ thick films can be significantly increased (almost doubled) compared to room temperature by using the thermoelectric cooling of a film sample.

The comparison of PL measurements results for both systems (single crystals and films) indicates the existence of some redistribution of intensity between the PL bands at low temperatures. In particular, for the investigated thick films, the PL intensities of E line related to the exciton recombination, as well as D1 band, corresponding to the recombination of DAPs involving shallow acceptor centers, are strongly decreased in comparison with the bulk crystal. Such a behavior is due to the fact that the $\mathrm{PbCdI}_{2}$ films exhibit much stronger structural heterogeneities than bulk $\mathrm{PbCdI}_{2}$ crystals of the same composition.

Analysis of the integral intensity of various PL bands showed that relative contribution of the surface states (STE and surface lead vacancies) to the PL spectrum at room temperature both for the $\mathrm{PbCdI}_{2}$ thick films investigated and for bulk nanostructured solid solutions is similar and is about $50 \%$.

The obtained results on the nature of the radiative recombination processes in thick $\mathrm{PbCdI}_{2}$ films make it possible to identify more clearly the features of the crystal and defect structure of the investigated films. The presence of the STE band both at low and at room temperature indicates the formation of small $\mathrm{PbI}_{2}$ NCLs in thick films with sizes comparable to the Bohr radius of a bulk crystal exciton $(1.9 \mathrm{~nm})$ [9]. As was noted earlier, direct observation of very small $\mathrm{PbI}_{2}$ NCLs in SEM images is obstructed by the presence of a thin layer of solvent on the surface of the thick films.

\section{Conclusions}

We report on a simple inexpensive method for preparing the $\mathrm{PbCdI}_{2}$ films which exhibit intense photoluminescence at both low and room temperatures similarly to the bulk $\mathrm{Pb}_{0.30} \mathrm{Cd}_{0.70} \mathrm{I}_{2}$ solid solutions. The structure and temperature dependence of the photoluminescence spectra of the films were analyzed. The radiative recombination processes in these films were compared to analogous processes in bulk nanostructured solid solutions. XRD measurements showed that $\mathrm{PbCdI}_{2}$ films grown by the conventional one-step deposition method are crystalline rather than amorphous. The analysis of their XRD spectrum indicates that the investigated alloys include different crystalline $\mathrm{CdI}_{2}$ and $\mathrm{PbI}_{2}$ phases. In particular, there are three polytypes of $\mathrm{CdI}_{2}$, namely, $2 \mathrm{H}, 4 \mathrm{H}$, and $12 \mathrm{R}$. At the same time, the dominant phase of $\mathrm{PbI}_{2}$ is $4 \mathrm{H}$ polytype.

It was shown that the PL spectrum of such films at $T=$ $10 \mathrm{~K}$ is formed by emission of free and bound excitons in $\mathrm{PbI}_{2}$ microcrystallites, recombination of self-trapped excitons in small $\mathrm{PbI}_{2}$ NCLs, and donor-acceptor pairs with the participation of the shallow acceptor centers as well as recombination of free electrons in the conduction band with the holes of deep acceptor centers. The emission of the films at room temperature is equally determined by the recombination of surface states, which are associated with both the formation of small $\mathrm{PbI}_{2}$ NCLs in $\mathrm{CdI}_{2}$ matrix and lead vacancies on these surfaces and intrinsic defects in 
microcrystallites. The obtained results indicate that $\mathrm{PbCdI}_{2}$ films can be considered as nanostructured film materials where in some crystal regions I-Cd-I layers are partially replaced by I-Pb-I layers. The sizes of these crystal regions can vary from several $\mathrm{nm}$ to several tens or hundreds of $\mathrm{nm}$ (submicron crystal regions). Here, there are also $\mathrm{PbI}_{2}$ crystal regions of several micron sizes. Thus, these films are very inhomogeneous. In our opinion, the investigated $\mathrm{PbCdI}_{2}$ thick films can be considered as very promising semiconductor materials suitable for future development on their basis of novel inexpensive effective scintillator detectors for biomedical and industrial applications.

\section{Conflicts of Interest}

The authors declare that they have no conflicts of interest.

\section{Acknowledgments}

This research has been supported by the National Academy of Sciences of Ukraine (Grants nos. BC-157-15 and B-146-15).

\section{References}

[1] H. Gleiter, "Nanostructured materials: state of the art and perspectives," Nanostructured Materials, vol. 6, no. 1-4, pp. 3-14, 1995.

[2] H. Gleiter, "Nanostructured Materials - Scientific Background and Technological Perspectives," Materials Science Forum, vol. 67, pp. 189-190, 1995.

[3] M. Velický, M. A. Bissett, C. R. Woods et al., "Photoelectrochemistry of Pristine Mono- and Few-Layer MoS2," Nano Letters, vol. 16, no. 3, pp. 2023-2032, 2016.

[4] E. D. Bourret-Courchesne, S. E. Derenzo, and M. J. Weber, "Semiconductor scintillators $\mathrm{ZnO}$ and $\mathrm{PbI}$ : Co-doping studies," Nuclear Instruments and Methods in Physics Research Section A: Accelerators, Spectrometers, Detectors and Associated Equipment, vol. 579, no. 1, pp. 1-5, 2007.

[5] S. E. Derenzo, E. Bourret-Courchesne, Z. Yan, G. Bizarri, A. Canning, and G. Zhang, "Experimental and theoretical studies of donor-acceptor scintillation from PbI2," Journal of Luminescence, vol. 134, pp. 28-34, 2013.

[6] P. J. Sellin, "Thick film compound semiconductors for X-ray imaging applications," Nuclear Instruments and Methods in Physics Research Section A: Accelerators, Spectrometers, Detectors and Associated Equipment, vol. 563, no. 1, pp. 1-8, 2006.

[7] K. Shah, R. Street, Y. Dmitriyev et al., "X-ray imaging with PbI2-based A-Si:H flat panel detectors," Nuclear Instruments and Methods in Physics Research Section A: Accelerators, Spectrometers, Detectors and Associated Equipment, vol. 458, no. 1-2, pp. 140-147, 2001.

[8] M. M. Marino, M. Sawamura, W. C. Ermler, and C. J. Sandroff, "Ab initio study of the geometry and electronic structure of lead iodide semiconductor clusters," Physical Review B: Condensed Matter and Materials Physics, vol. 41, no. 2, pp. 1270-1273, 1990.

[9] M. S. Furyer, P. A. Skubenko, P. M. Bukivskij et al., "Study of the photoluminescence and photoelectric properties of $\mathrm{Pb} 1-$ XCdXI2 alloys," Journal of Applied Physics, vol. 108, no. 10, Article ID 103711, 2010.
[10] Y. P. Gnatenko, P. M. Bukivskij, Y. P. Piryatinski, A. P. Bukivskii, P. A. Skubenko, and R. V. Gamernyk, "Time-resolved photoluminescence spectroscopy of excitons in layered semiconductor PbI2 nanoclusters," Journal of Applied Physics, vol. 112, no. 9, Article ID 093708, 2012.

[11] A. P. Bukivskii, Y. P. Gnatenko, Y. P. Piryatinskii, and R. V. Gamernyk, "Nature of radiative recombination processes in layered semiconductor $\mathrm{PbCdI} 2$ nanostructural scintillation material," Journal of Luminescence, vol. 185, pp. 83-91, 2017.

[12] Y. P. Gnatenko, M. S. Fur'yer, P. M. Bukivskij, P. A. Skubenko, Y. P. Piryatinski, and R. V. Gamernyk, "Photoluminescence properties of layered $\mathrm{Pb}_{1-x} \mathrm{Cd}_{x I 2}$ solid solutions," Ukrainian Journal of Physics, vol. 56, no. 10, pp. 1015-1020, 2011.

[13] A. P. Bukivskii, A. O. Sofiienko, V. Y. Degoda, R. V. Gamernyk, and Y. P. Gnatenko, "Photoluminescence and X-ray luminescence of $\mathrm{Pb} 0.30 \mathrm{Cd} 0.70 \mathrm{I} 2$ solid solutions. Comparative study," Materials Science in Semiconductor Processing, vol. 67, pp. 2832, 2017.

[14] A. P. Bukivskii and Y. P. Gnatenko, "Study of the photoluminescence kinetics of heterogeneous nanostructured $\mathrm{Pb} 0.30 \mathrm{Cd} 0.70 \mathrm{I} 2$ solid solutions," Materials Chemistry and Physics, vol. 199, pp. 577-584, 2017.

[15] R. Ahuja, H. Arwin, A. F. Da Silva et al., "Electronic and optical properties of lead iodide," Journal of Applied Physics, vol. 92, no. 12, pp. 7219-7224, 2002.

[16] I. C. Schlüter and M. Schlüter, "Electronic structure and optical properties of PbI2," Physical Review B: Condensed Matter and Materials Physics, vol. 9, no. 4, pp. 1652-1663, 1974.

[17] M. Watanabe, A. Ishibashi, and T. Hayashi, "Free and localized exciton luminescence in $\mathrm{Pb}_{1-x} \mathrm{Cd}_{x I 2}$ mixed crystals," Journal of Luminescence, vol. 48-49, no. 1, pp. 87-90, 1991.

[18] P. Luo, W. Xia, S. Zhou et al., "Solvent Engineering for AmbientAir-Processed, Phase-Stable CsPbI3 in Perovskite Solar Cells," The Journal of Physical Chemistry Letters, vol. 7, no. 18, pp. 36033608,2016

[19] W. M. Sears, M. L. Klein, and J. A. Morrison, "Polytypism and the vibrational properties of PbI2," Physical Review B: Condensed Matter and Materials Physics, vol. 19, no. 4, pp. 23052313, 1979.

[20] F. Lévy, A. Mercier, and J.-P. Voitchovsky, "Band-edge photoluminescence of PbI2," Solid State Communications, vol. 15, no. 5, pp. 819-822, 1974.

[21] D. H. Jaw, J. R. Chang, and Y. K. Su, "Observation of selforganized superlattice in AlGaInAsSb pentanary alloys," Applied Physics Letters, vol. 82, no. 22, pp. 3883-3885, 2003.

[22] L. S. Vavilova, V. A. Kapitonov, A. V. Murashova et al., "Spontaneously assembling periodic composition-modulated InGaAsP structures," Semiconductors, vol. 33, no. 9, pp. 1010-1012, 1999.

[23] G. B. Stringfellow, "Spinodal decomposition and clustering in III/V alloys," Journal of Electronic Materials (JEM), vol. 11, no. 5, pp. 903-918, 1982.

[24] J. Androulakis, C.-H. Lin, H.-J. Kong et al., "Spinodal decomposition and nucleation and growth as a means to bulk nanostructured thermoelectrics: Enhanced performance in $\mathrm{Pb}_{1-x} \mathrm{Sn}_{x} \mathrm{Te}-$ PbS," Journal of the American Chemical Society, vol. 129, no. 31, pp. 9780-9788, 2007.

[25] B. Kumar and N. Sinha, "Micromorphology of pure and PbI2doped CdI2 dendritic single crystals," Crystal Research and Technology, vol. 40, no. 9, pp. 887-892, 2005.

[26] A. E. Dugan and H. K. Henisch, "Defect energy-level structure of PbI2 single crystals," Physical Review A: Atomic, Molecular and Optical Physics, vol. 171, no. 3, pp. 1047-1051, 1968. 
[27] P. Gu, M. Watanabe, and T. Hayashi, "Electronic States and Energy Transfer in PbI2Clusters Dispersed in CdI2Crystals," Journal of the Physical Society of Japan, vol. 64, no. 11, pp. 44504460, 1995.

[28] I. Baltog, M. Baibarac, and S. Lefrant, "Quantum well effect in bulk PbI2 crystalsrevealed by the anisotropy of photoluminescence and Raman spectra," Journal of Physics: Condensed Matter, vol. 21, no. 2, Article ID 025507, 2009.

[29] I. Dag and E. Lifshitz, "Dynamics of recombination processes in pbli nanocrystals embedded in porous silica films," The Journal of Physical Chemistry C, vol. 100, no. 21, pp. 8962-8972, 1996.

[30] E. Lifshitz, L. Bykov, M. Yassen, and Z. Chen-Esterlit, "The investigation of donor and acceptor states in nanoparticles of the layered semiconductor PbI2," Chemical Physics Letters, vol. 273, no. 5-6, pp. 381-388, 1997.

[31] E. Lifshitz, M. Yassen, L. Bykov, and I. Dag, "Continuous photoluminescence, time resolved photoluminescence and optically detected magnetic resonance measurements of PbI2 nanometer-sized particles, embedded in $\mathrm{SiO} 2$ films," Journal of Luminescence, vol. 70, no. 1-6, pp. 421-434, 1996.

[32] I. Baltog, I. Piticu, M. Constantinescu, C. Ghita, and L. Ghita, "Optical investigations of PbI2 single crystals after thermal treatment," Physica Status Solidi (a) - Applications and Materials Science, vol. 52, no. 1, pp. 103-110, 1979.

[33] E. Lifshitz, M. Yassen, L. Bykov, I. Dag, and R. Chaim, "Photodecomposition and regeneration of $\mathrm{PbI} 2$ nanometer-sized particles, embedded in porous silica films," The Journal of Physical Chemistry C, vol. 99, no. 4, pp. 1245-1250, 1995.

[34] M. H. Nayfeh, N. Rigakis, and Z. Yamani, "Photoexcitation of $\mathrm{Si}-\mathrm{Si}$ surface states in nanocrystallites". 

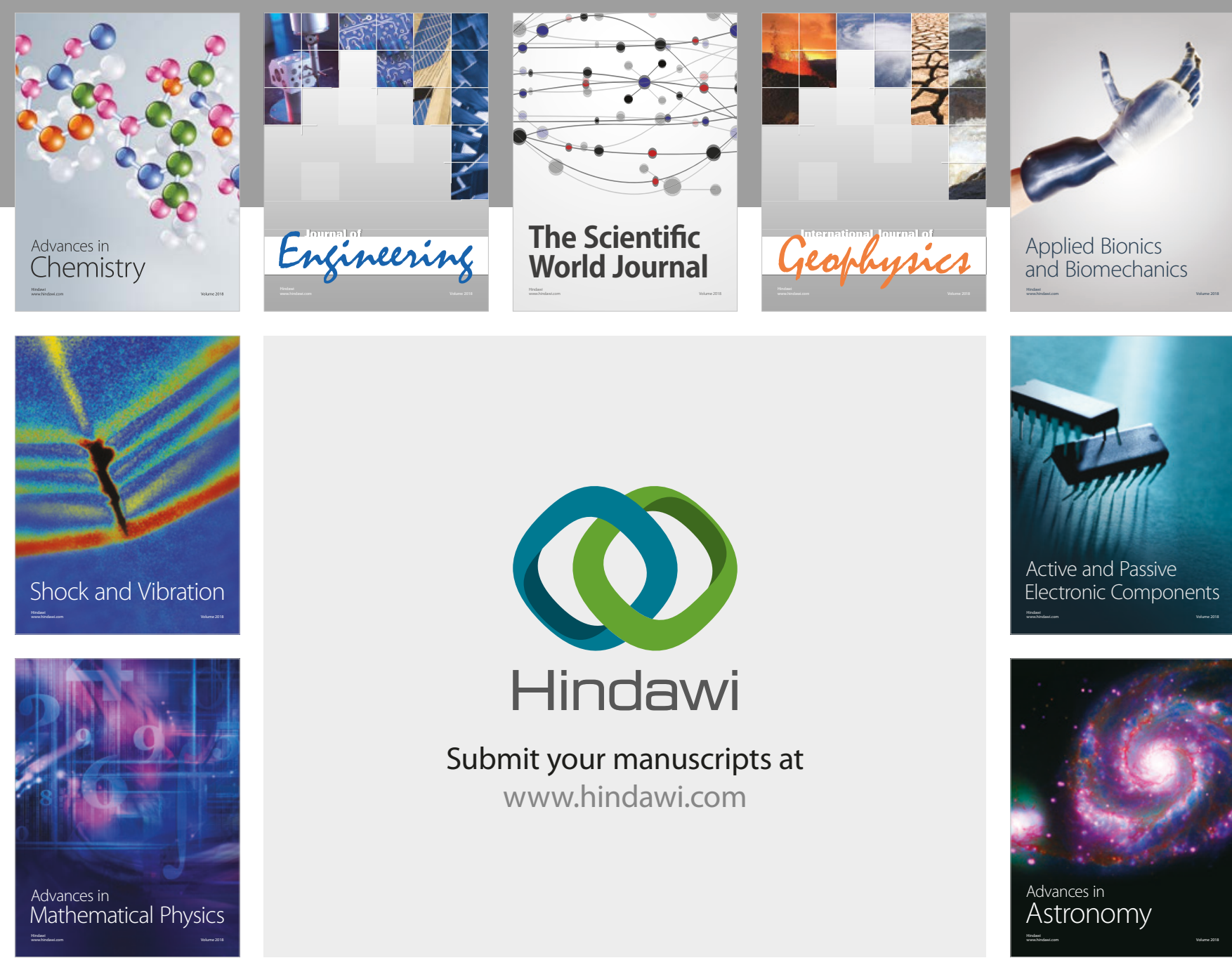

Submit your manuscripts at

www.hindawi.com

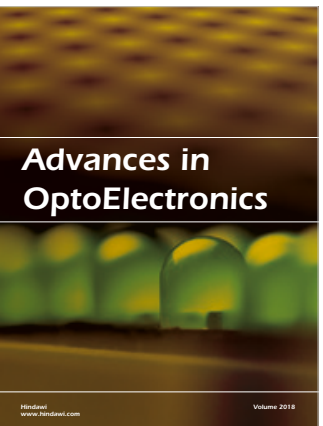

\section{Rotcting Machinery}
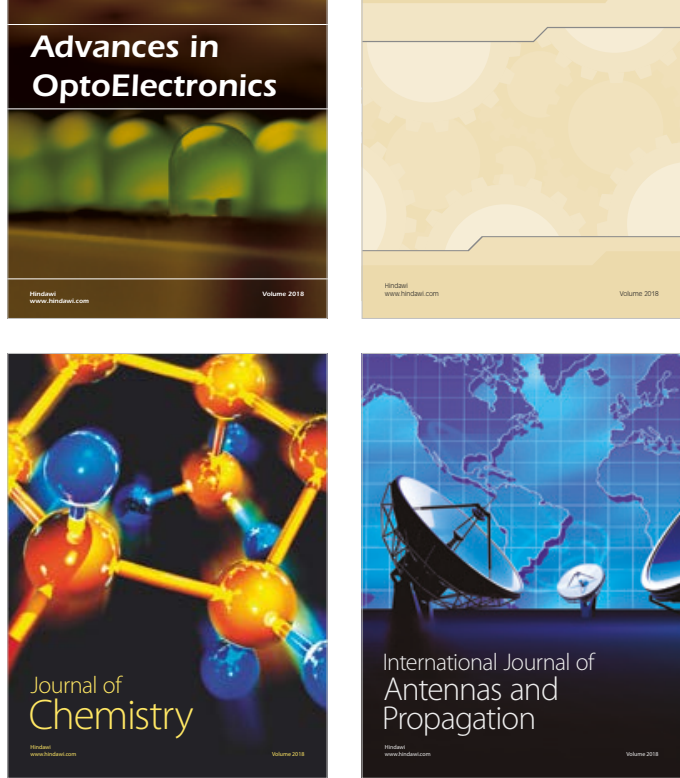

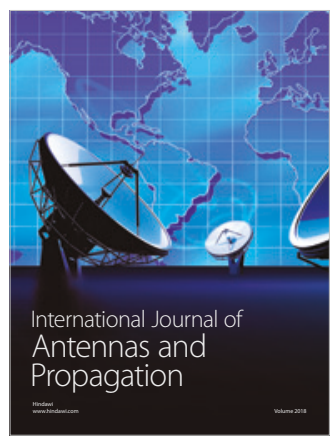

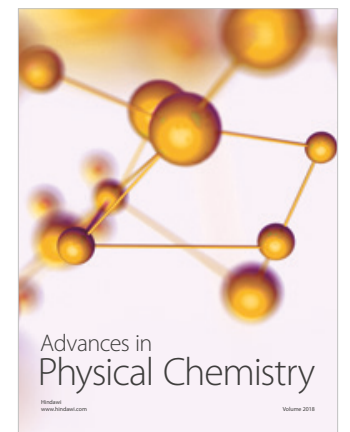

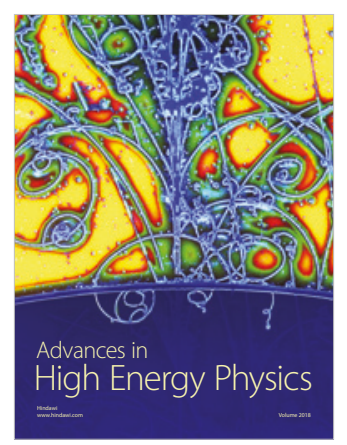

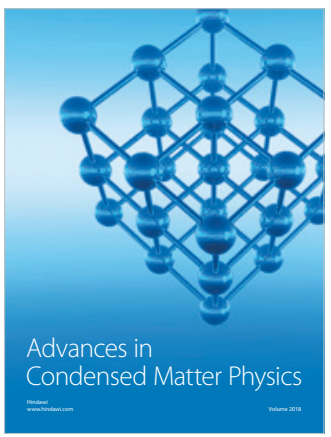

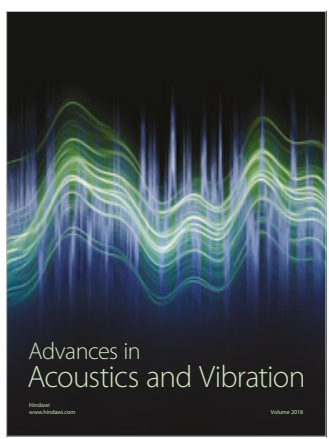

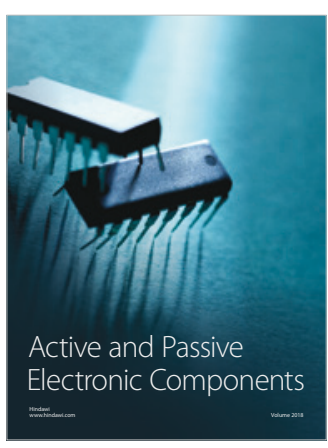
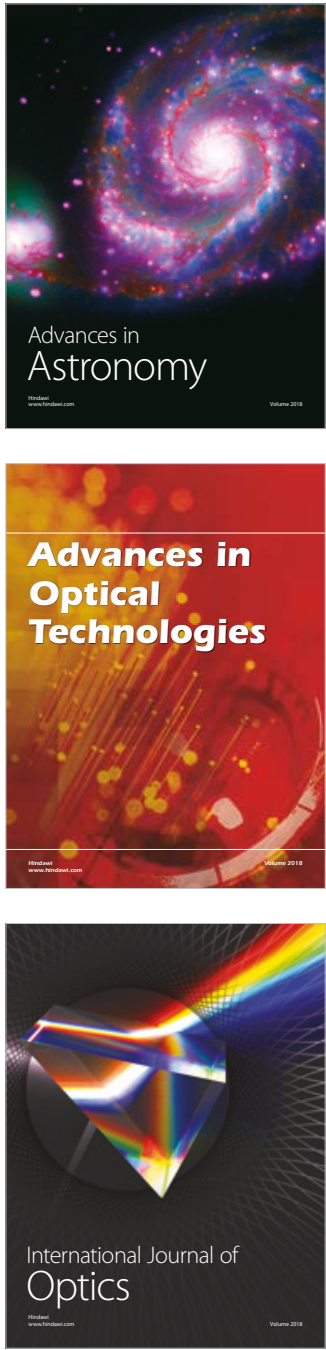